\title{
Möbius Stanchion Systems
}

\section{Lucas Isenmann, Timothée Pecatte}

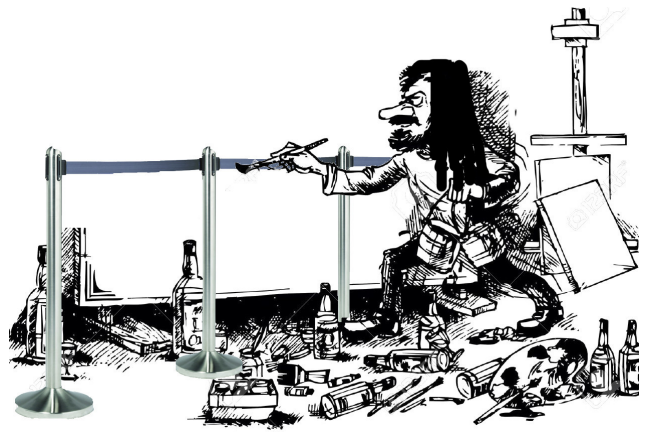




\section{Stanchion system}

What are stanchions?

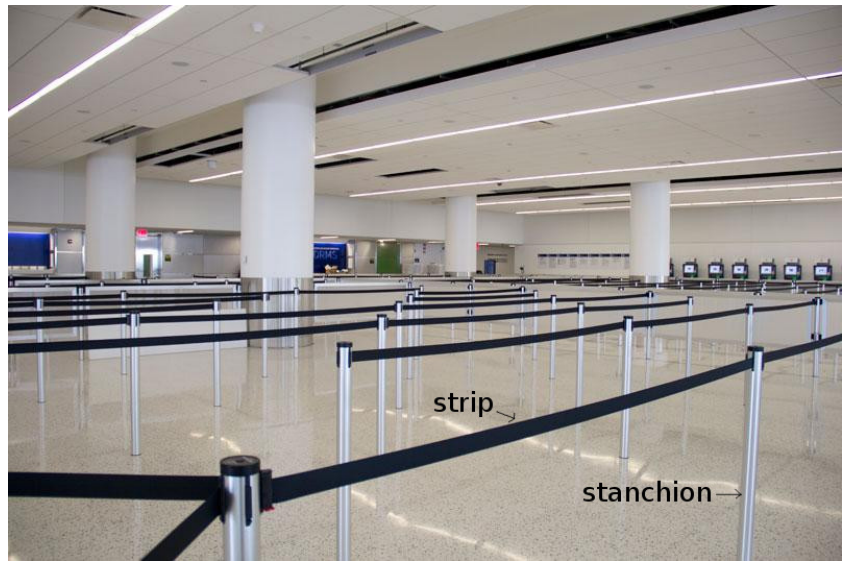




\section{Stanchion painting problem}

Problem: paint both sides of every strips of a stanchion system. 


\section{Stanchion painting problem}

Problem: paint both sides of every strips of a stanchion system.

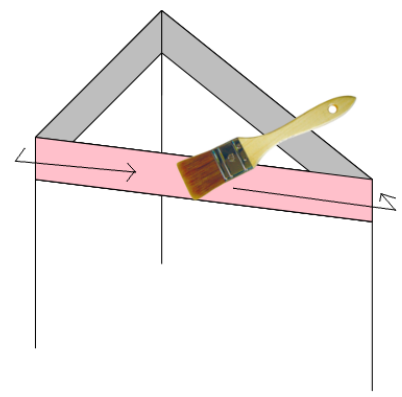




\section{Stanchion painting problem}

Problem: paint both sides of every strips of a stanchion system.

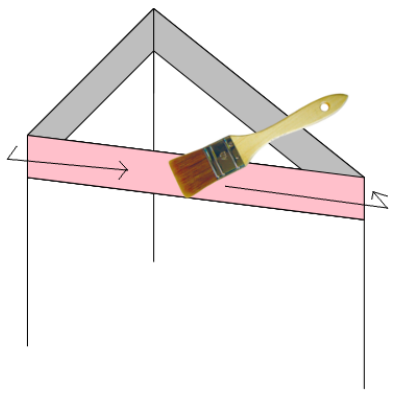

Condition: we do not want to lift up the brush. 


\section{Stanchion painting problem}

Solution: twist a strip!

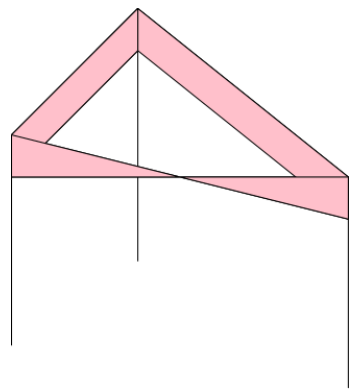




\section{Stanchion painting problem}

Solution: twist a strip!

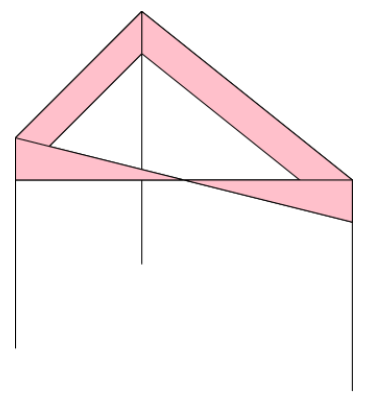

The painter can paint without lifting up the brush! 


\section{Modelisation}

A ribbon graph
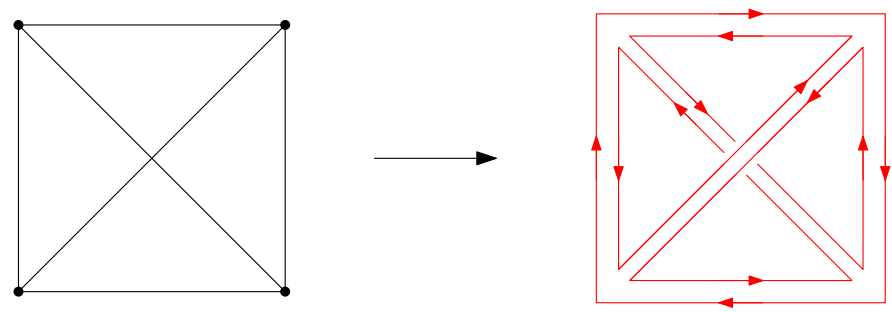


\section{Modelisation}

A ribbon graph
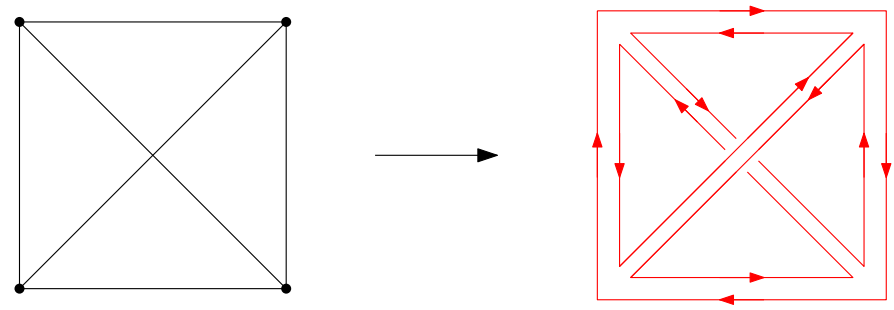

With twisted strips:

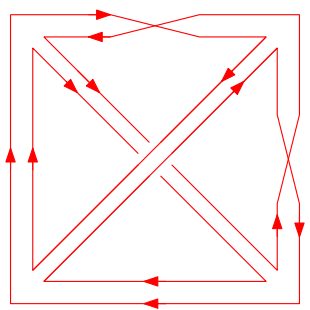




\section{Modelisation}

Combinatorial map: $\iota, \alpha$ and $\beta$ are involution on quarter-edges.

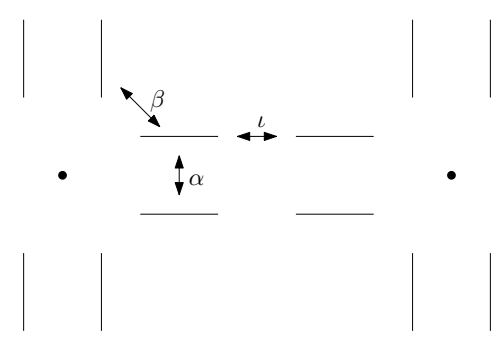




\section{Modelisation}

Combinatorial map: $\iota, \alpha$ and $\beta$ are involution on quarter-edges.

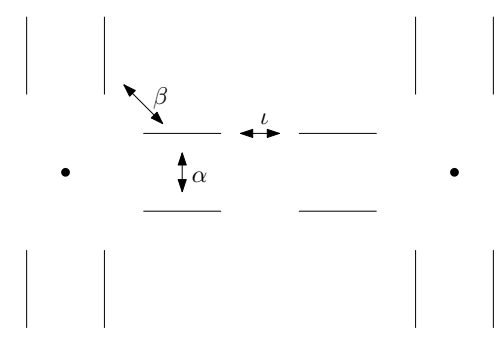

With twisted strips:

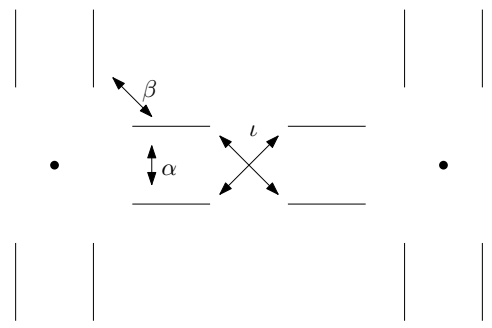




\section{Question}

For this graph, the walk of the brush makes 2 cycles:

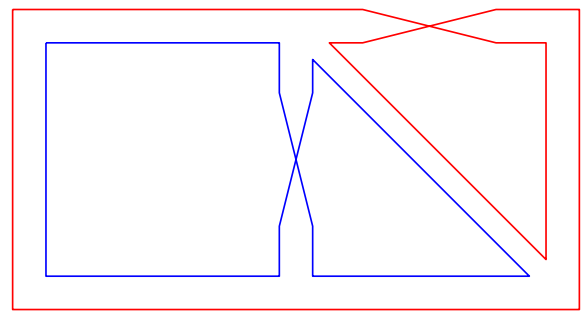




\section{Question}

For this graph, the walk of the brush makes 2 cycles:

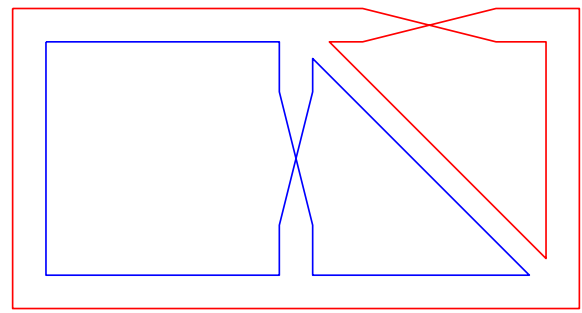

How to twist strips so that there is only one cycle?

A solution $=$ Möbius stanchion system 
Can you twist some edges to solve the problem for this graph?

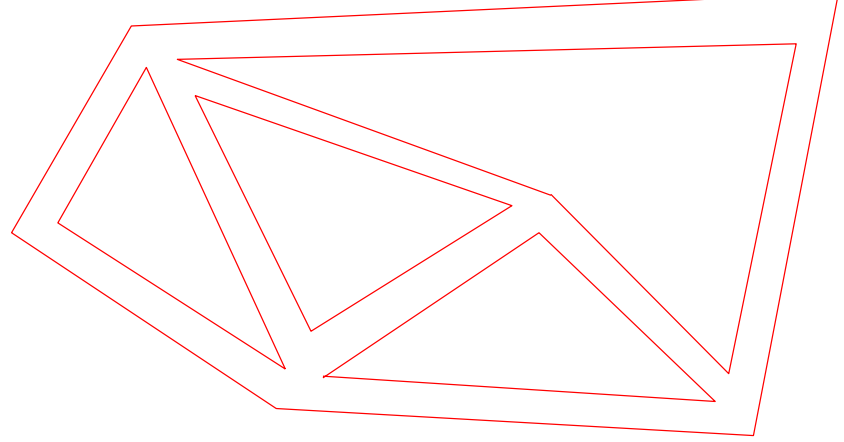




\section{Your turn!}

Can you twist some edges to solve the problem for this graph?

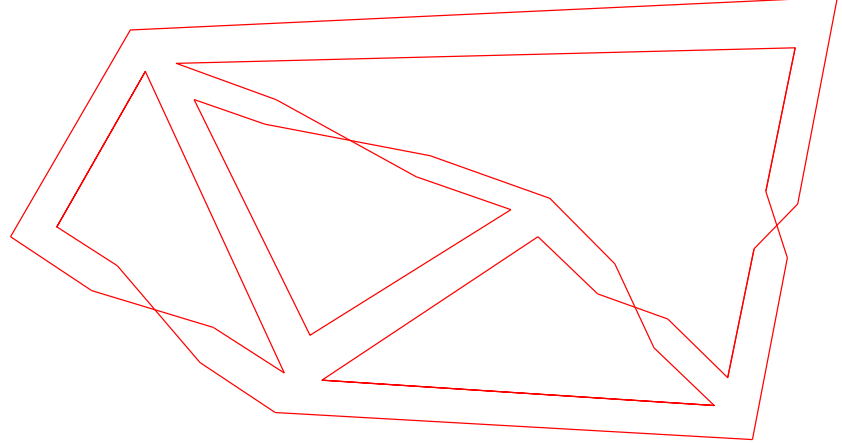




\section{Your turn!}

Can you twist some edges to solve the problem for this graph?

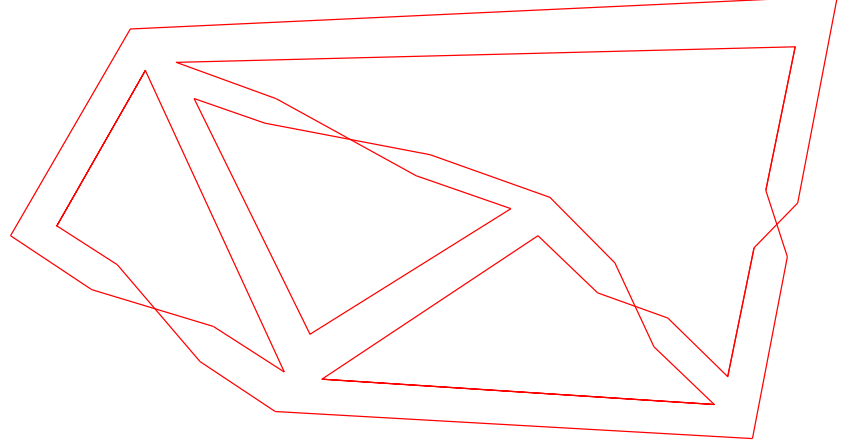

Claim: 4 twists is the minimum 


\section{Dual graph}

Here, the minimum number is the number of interior faces.

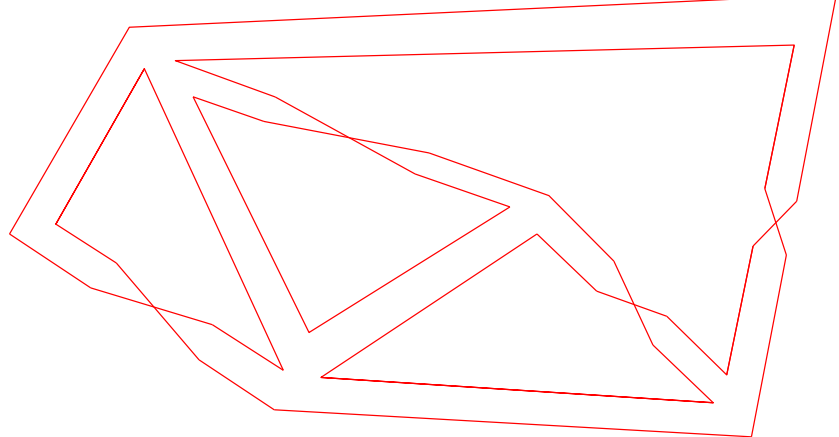




\section{Dual graph}

Here, the minimum number is the number of interior faces.

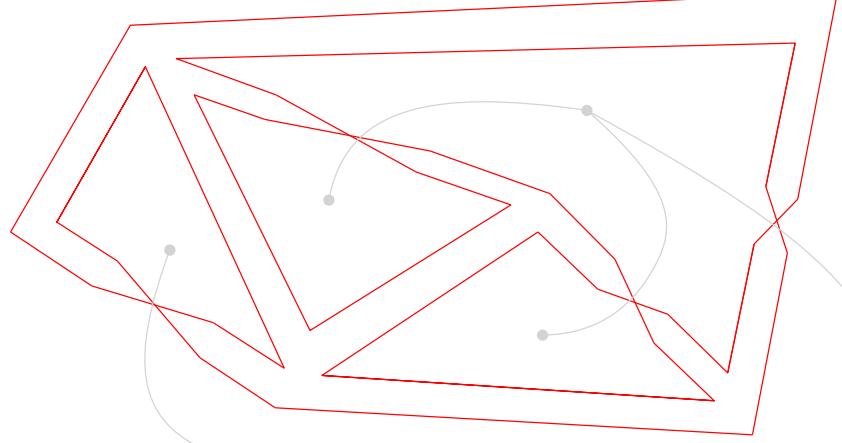

Twisted strips connect faces $\rightarrow$ subgraph of the dual graph 


\section{Necessary condition}

In a solution, all faces should be connected = connected spanning subgraph of the dual graph

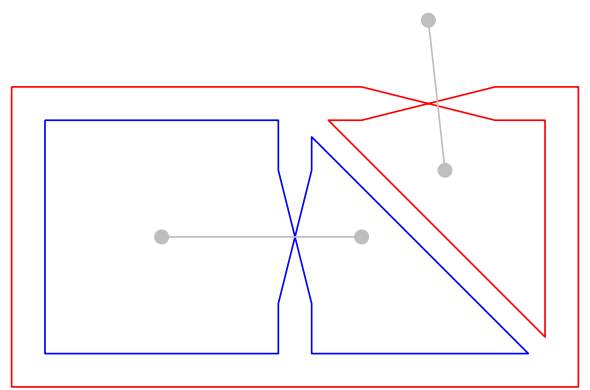




\section{Minimal solutions}

A spanning tree of the dual graph gives a solution

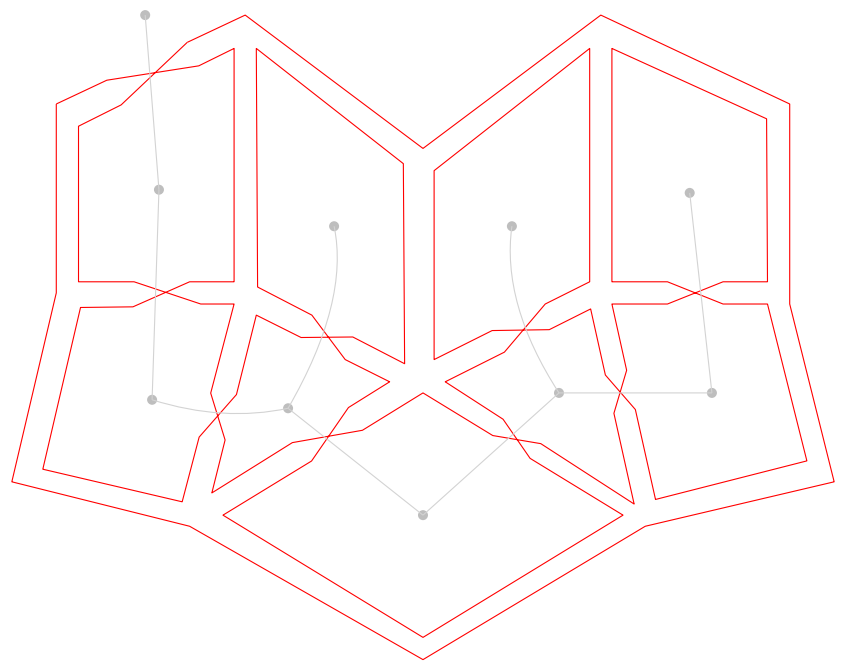




\section{Minimal solutions}

\section{Theorem}

Spanning trees of the dual are the minimal solutions with $f-1$ twisted edges ( $f$ is the number of faces). Therefore the painter needs at least to twist as much as there are interior faces. 
Are there other solutions? 
Are there other solutions?

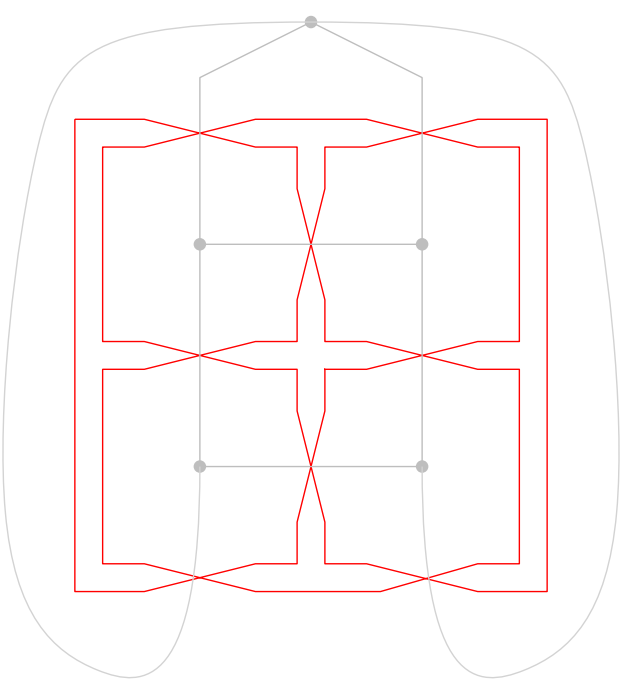

Yes!

$13 / 25$ 


\section{How to get other solutions}

Start with a solution given by a spanning tree 
Start with a solution given by a spanning tree and try to modify it using some preserving rules. 
Start with a solution given by a spanning tree and try to modify it using some preserving rules.

Two elementary operations :

- single twist 


\section{Start with a solution given by a spanning tree and try to modify it using some preserving rules.}

Two elementary operations :

- single twist

- double twist 


\section{Elementary operations}

Single twist $=$ twist an edge 


\section{Elementary operations}

Single twist $=$ twist an edge

Some edges are twistable $\rightarrow$ another solution
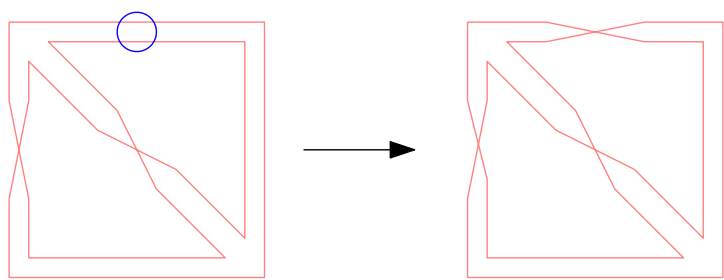


\section{Elementary operations}

Single twist $=$ twist an edge

Some edges are twistable $\rightarrow$ another solution
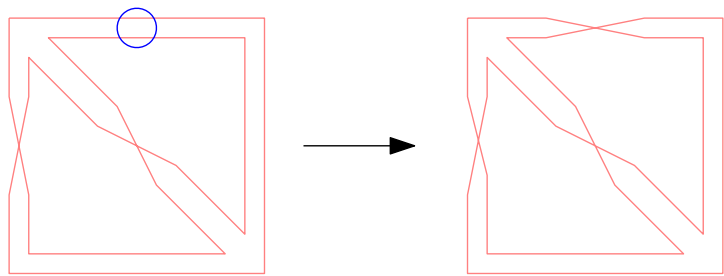

Some others are not $\rightarrow$ not a solution
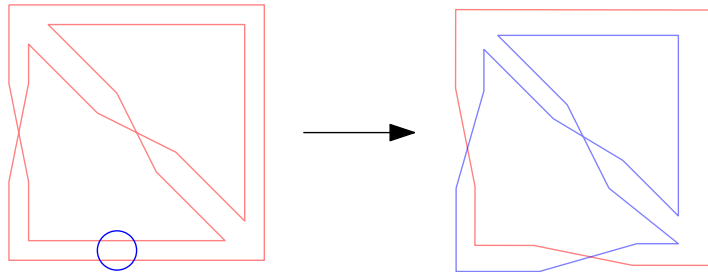


\section{Elementary operations}

The good edges are crossed two-ways

Single twist of a good edge $\rightarrow$ still a solution 


\section{Elementary operations}

The good edges are crossed two-ways

Single twist of a good edge $\rightarrow$ still a solution

Example:

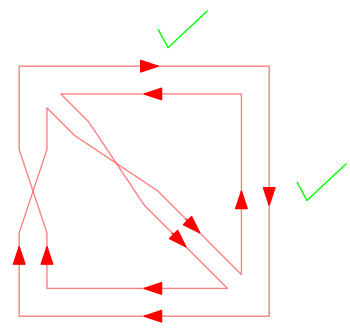




\section{Elementary operations}

Double twist $=$ twist two edges simultaneously 


\section{Elementary operations}

Double twist $=$ twist two edges simultaneously

Some pair of edges seems to be twistable:
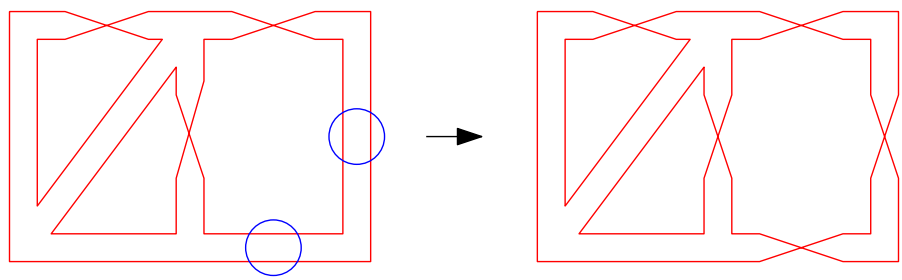


\section{Elementary operations}

Double twist $=$ twist two edges simultaneously

Some pair of edges seems to be twistable:
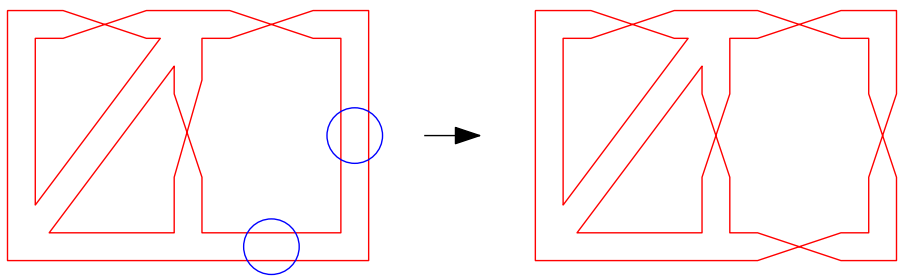

But some others not:
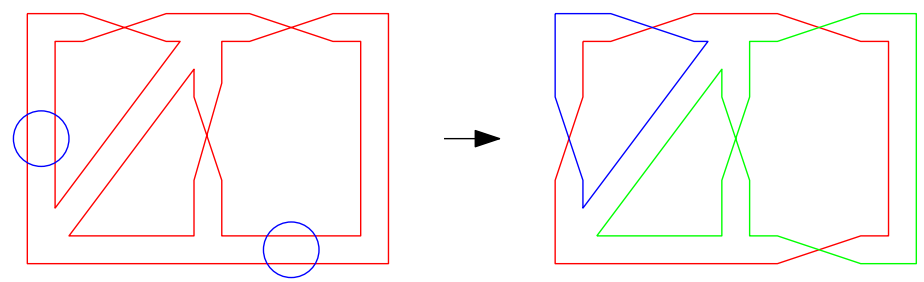


\section{Elementary operations}

A good pair of strips need the following connexions: 


\section{Elementary operations}

A good pair of strips need the following connexions:

When twisted:
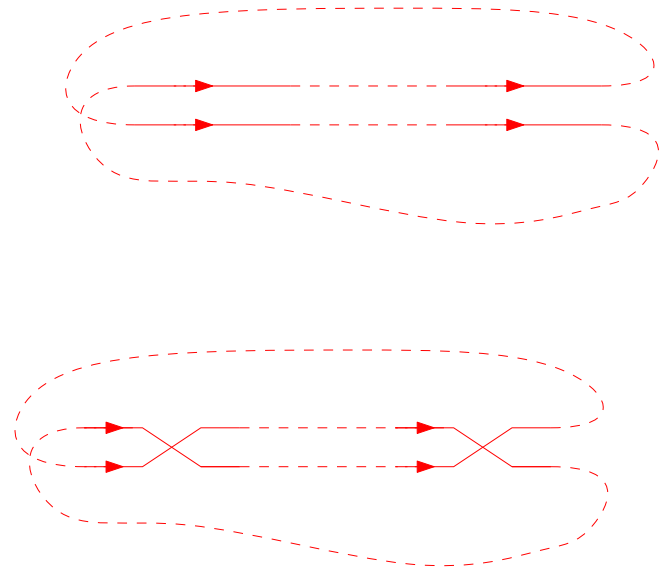

This double twist still gives a solution. 


\section{Elementary operations}

A good pair of strips need the following connexions:

Example:

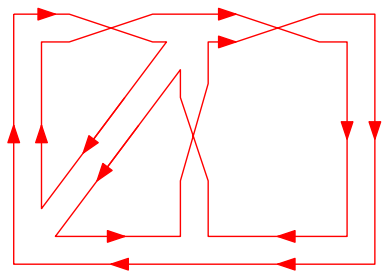




\section{Example}

Let's start with this spanning tree solution:

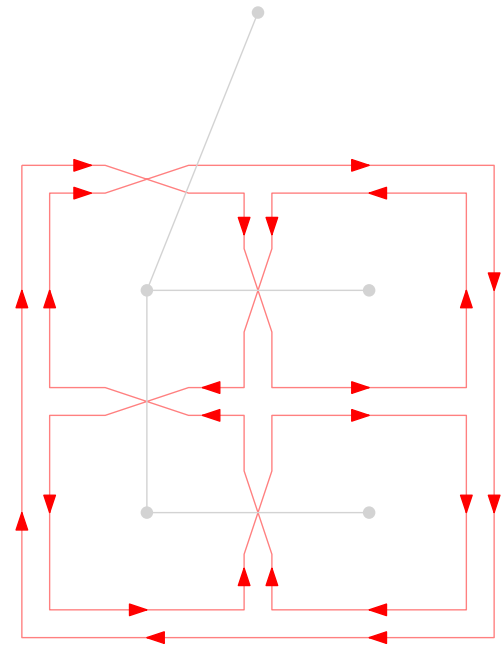




\section{Example}

We can single twist this strip:

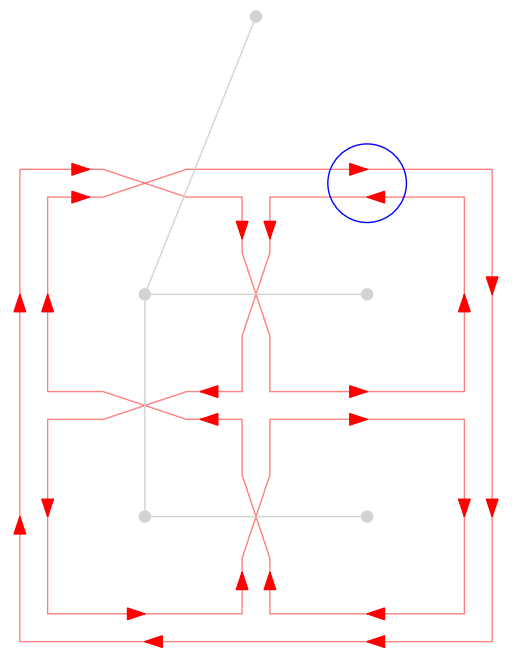




\section{Example}

We get a new solution:

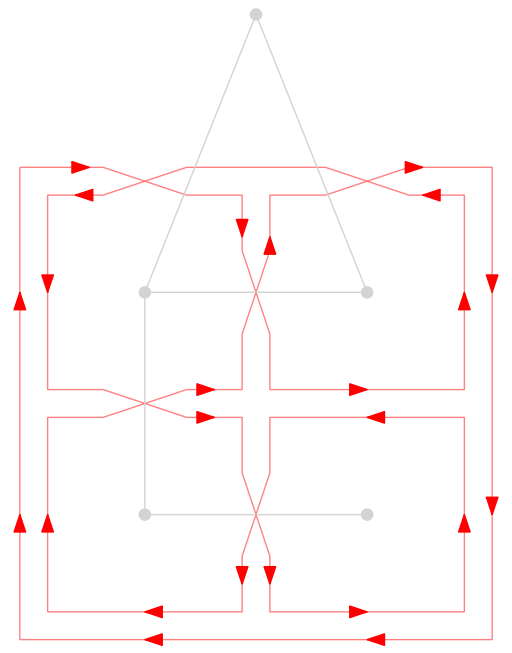




\section{Example}

We can single twist this strip:

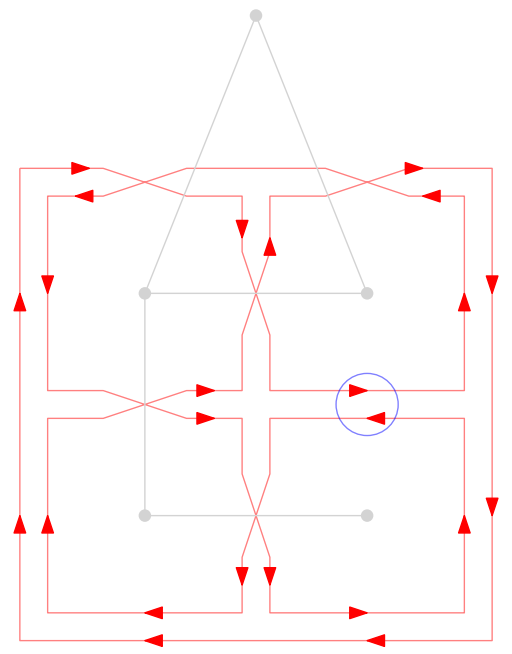




\section{Example}

Another new solution:

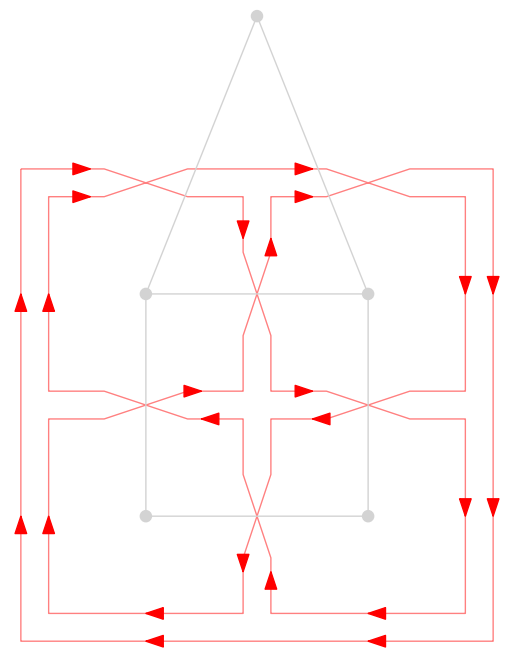




\section{Example}

We can double twist this pair:

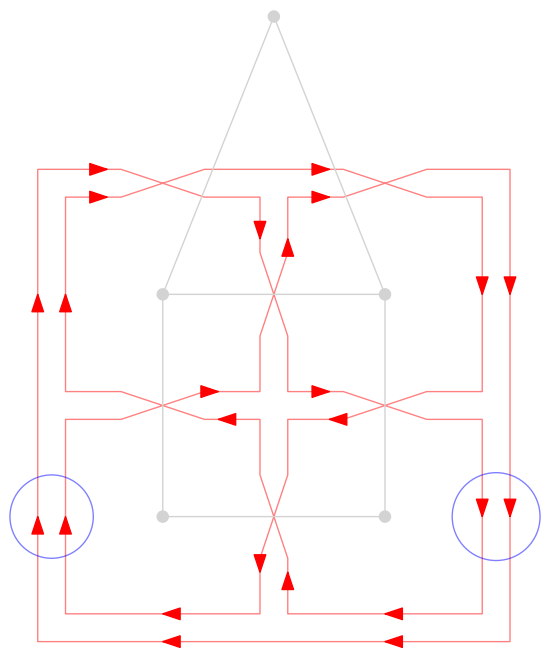




\section{Example}

Another solution:

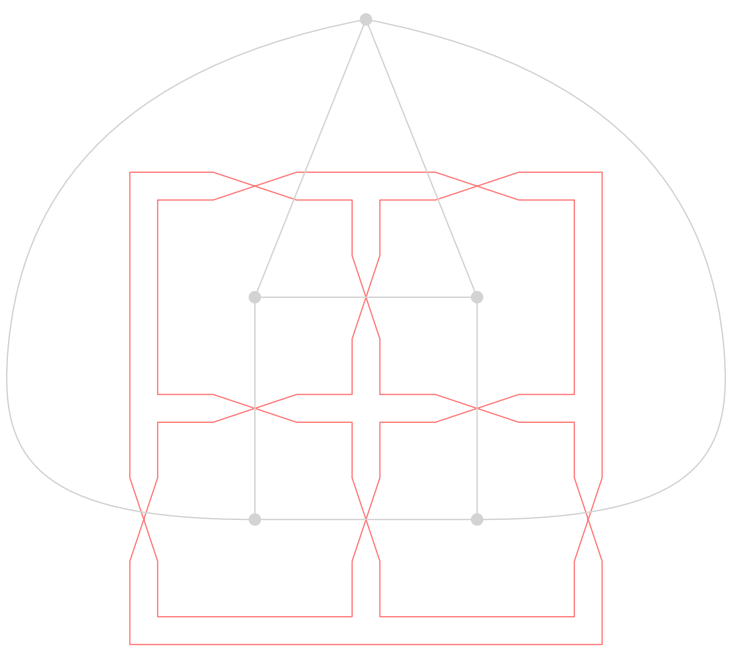




\section{Elementary operations}

\section{Theorem}

From a solution we can get any other solution by applying successive single and double twists.

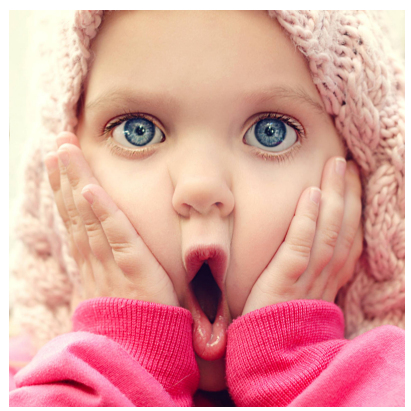




\section{Sketch of proof by induction}

Ok for trees ( $f-1$ twisted edges). 


\section{Sketch of proof by induction}

Take a solution $\geq f$ twisted edges. 


\section{Sketch of proof by induction}

Take a solution $\geq f$ twisted edges.

Suppose: no feasible simple twist and double twist 


\section{Sketch of proof by induction}

Take a solution $\geq f$ twisted edges.

Suppose: no feasible simple twist and double twist

$\Rightarrow$ no twisted two-ways edge, no good pair of twisted edges 


\section{Sketch of proof by induction}

Take a solution $\geq f$ twisted edges.

Suppose: no feasible simple twist and double twist

$\Rightarrow$ no twisted two-ways edge, no good pair of twisted edges

not a tree $\Rightarrow \exists$ minimal cycle $C$ 


\section{Sketch of proof by induction}

Take a solution $\geq f$ twisted edges.

Suppose: no feasible simple twist and double twist

$\Rightarrow$ no twisted two-ways edge, no good pair of twisted edges

not a tree $\Rightarrow \exists$ minimal cycle $C$

it cannot be odd

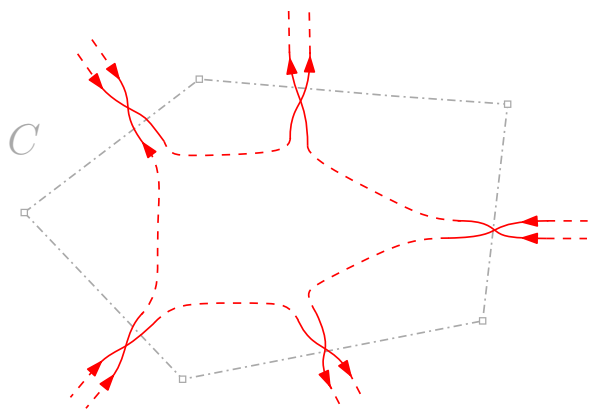




\section{Sketch of proof by induction}

Take a solution $\geq f$ twisted edges.

Suppose: no feasible simple twist and double twist

$\Rightarrow$ no twisted two-ways edge, no good pair of twisted edges

not a tree $\Rightarrow \exists$ minimal cycle $C$

it cannot be even

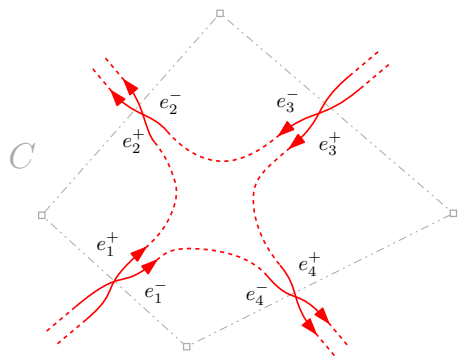

cyclic order : $\left[e_{1}^{+}, e_{2}^{+}, e_{2}^{-}, e_{1}^{-}\right],\left[e_{2}^{+}, e_{3}^{+}, e_{3}^{-}, e_{2}^{-}\right], \ldots$

$\left[e_{1}^{+}, \ldots, e_{4}^{+}, e_{1}^{+}, e_{1}^{-}, e_{4}^{-}, \ldots, e_{1}^{-}\right]$gives a contradiction 


\section{Embeddings and paintings}

\section{Theorem}

Let $G$ be a planar graph. The Möbius stanchions systems of $G$ are independent of the chosen embedding for $G$ in the plane.
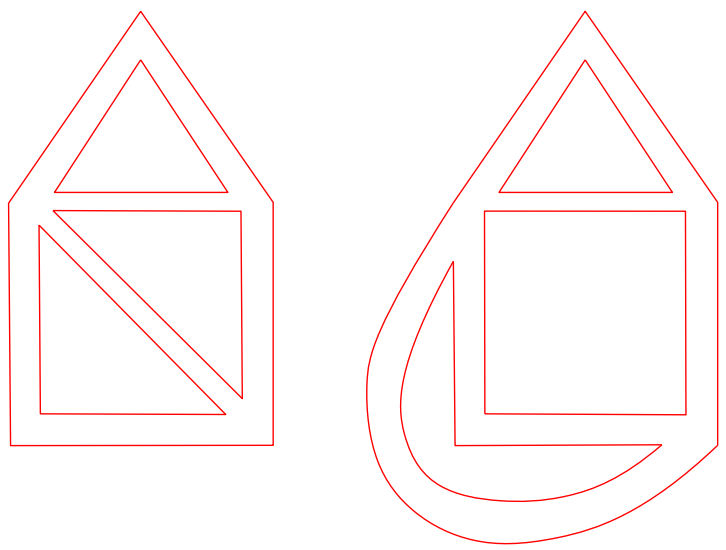

Figure: two non-isomorphic embeddings 


\section{Sketch of proof}

Planar 3-connected $\Rightarrow$ only one embedding. 


\section{Sketch of proof}

Planar 3-connected $\Rightarrow$ only one embedding.

Not 3-connected : swap and flip operations connect all the embeddings
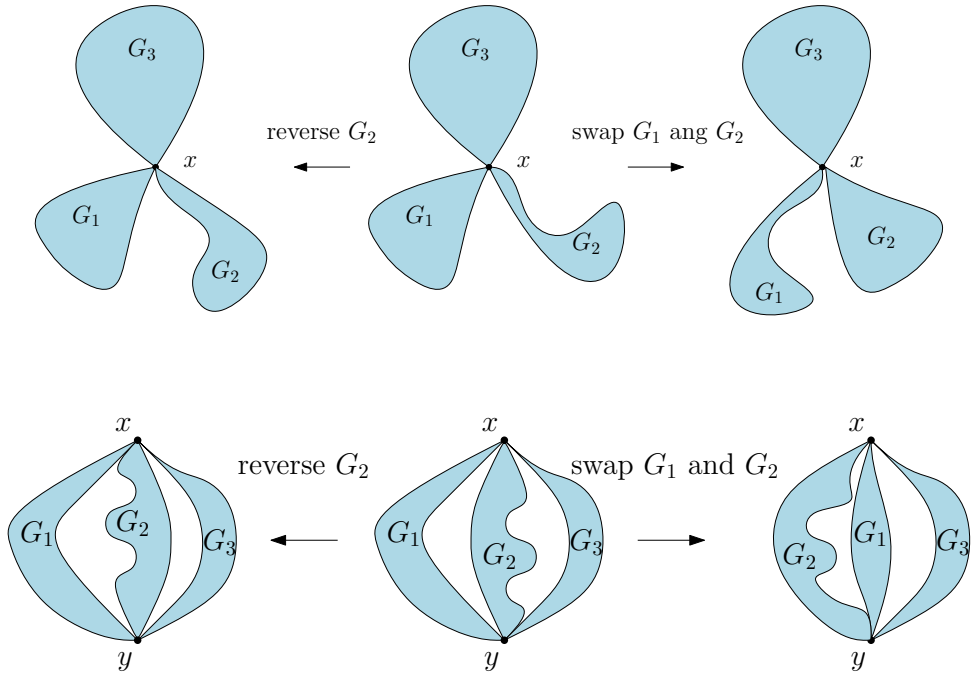


\section{Open questions}

- diameter of the MSS's graph? 


\section{Open questions}

- diameter of the MSS's graph?

- what are the maximal solutions? 


\section{Open questions}

- diameter of the MSS's graph?

- what are the maximal solutions?

- solutions are independent from the embedding : combinatorial caracterisation? 


\section{Open questions}

- diameter of the MSS's graph?

- what are the maximal solutions?

- solutions are independent from the embedding : combinatorial caracterisation?

- are the results the same on the torus? 


\section{Open questions}

- diameter of the MSS's graph?

- what are the maximal solutions?

- solutions are independent from the embedding : combinatorial caracterisation?

- are the results the same on the torus?

- how to generalize to higher dimensions? 


\section{Open questions}

- diameter of the MSS's graph?

- what are the maximal solutions?

- solutions are independent from the embedding : combinatorial caracterisation?

- are the results the same on the torus?

- how to generalize to higher dimensions?

- how can it help to understand unicellular embeddings? 
Thank you for your attention!

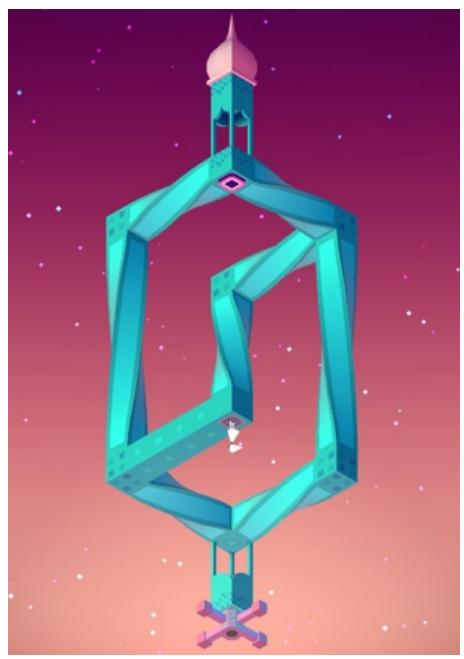

